\title{
Automated Bare Board Defect Detection using Pattern Subtraction Technique
}

\author{
Jithendra P.R. Nayak \\ Research Scholar, \\ GSSSIETW, Mysuru, \\ Karnataka, India
}

\author{
Parameshachari B.D., PhD \\ Department of TCE, \\ GSSSIETW, Mysuru, \\ Karnataka, India
}

\begin{abstract}
A Bare board or printed circuit board is the mechanical support that connects the electrical equipment using advanced means, track or signal tracked on copper sheets attached to a continuous substrate. Automated testing of PCBs serves a traditional purpose in computer technology. Aim is to free human inspectors from the inefficient and tedious task of detecting those defects in PCBs that could lead to failure. By comparing a typical PCB image with a faulty PCB image, using a simple extraction algorithm that can highlight major problem areas. Then perform a different image processing function such as blocking the image and filtering the image to remove unwanted edges and sound present in the wrong image and also the effect of noise on the PCB image is eliminated. This method should detect which method is appropriate to obtain the wrong image. Finally, segmentation is

used to identify the source of the six various defects such as under etch, mouse bite, pin hole, missing hole, open rotation and short circuit.
\end{abstract}

\section{Keywords}

Image Processing, Printed Circuit Board, Defect Classification

\section{INTRODUCTION}

Electrical and light detection are two types of printed circuit board quality tests. And visual-based visual processing technology has always been one of the hottest aspects of research on the benefits of offline communication, speed, accuracy, and the ability to combat distractions.

A visual inspection system of printed circuit board images can detect that breakdown as a bad idling area, even the point of cracking of solder, copper and platinum, short circuit etc using digital image processing and visual technology.

Image processing has two purposes, one to produce images that are more relevant to human identification and observation, the other one is to recognize and analyze images automatically.

The program includes a photo acquisition program, image enhancement, edge acquisition, feature detection. trying to use an extraction method to perform feature detection of printed circuit boards. The program aims to simplify real-world tasks that can provide the basis for online, accurate and fast circuit board acquisition

PCB defects are severely lost or additional elements on the board. The PCB errors or defects can be divided into two groups; functional disability and cosmetic disability.

Active disability can kill regional activities while cosmetic disability affects the appearance of the regional board but can also affect the performance of the circuit in the long run.

The PCB manufacturing process is dependent on mechanical and chemical actions that can damage the intended structure.
Typically, different PCB defects such as cracking, under etch, open circuit, mouse bite and pin-hole occur during production. The types of defects in single-phase PCBs are shown in Table 1.

Table 1. Defects on single layer Bare PCB

\begin{tabular}{|l|c|}
\hline No. & Defect \\
\hline 1 & Breakout \\
\hline 2 & Pin-hole \\
\hline 3 & Open circuit \\
\hline 4 & Under etch \\
\hline 5 & Mouse-bite \\
\hline 6 & Missing Conductor \\
\hline 7 & Spur \\
\hline 8 & Short \\
\hline 9 & Wrong Size Hole \\
\hline 10 & Conductor Too Close \\
\hline 11 & Spurious Copper \\
\hline 12 & Excessive Short \\
\hline 13 & Missing Hole \\
\hline 14 & Over Etch \\
\hline
\end{tabular}

\section{LITERATURE SURVEY}

PCB defect detection schemes generally uses the Machine learning and image processing techniques to find out the defects and to classify them. To find out the PCB errors and also to classify these errors, image processing can be used. Dave et al introduced a PCB error detecting system which uses image processing to find the defects in PCBs. The errors such as open circuits or missing holes can be found using this method [8]. Wu et al proposed a new visual inception technique which automatically detects errors in PCBs. The PCB template image is used to subtract with the PCB images which find the defects in the PCBs using the difference in the images.

The MATLAB based image processing technique is used by Kamalpreet et al to detect all the possible defects in PCBs. In this method, 14 possible defects are grouped into 5 groups [4]. Image processing methods and a morphological based algorithm of image segmentation is used by Putera et al to classify the PCB defects to find the defects and classify them. Also by using this system this method can detect and classify the defects on single layered bar PCBs [9]. The new algorithm based on wavelet, which automatically finds the image difference to find the defects on the PCBs is presented by Ibrahim et al . When compared to the previous traditional methods this scheme is very much efficient [10].

To improve the image processing technique's efficiency and the accuracy, few research works used machine learning techniques. Srimani et al introduced a new hybrid technique using soft computing methods to find the defects in PCBs and 
classify them. In this method, a neural network classifier and feature selection is achieved by using an adaptive genetic algorithm [11]. A new method which uses the data mining approach has been developed by Kusiak et al [12] , to overcome the issues due to the occurrence of solder-balls in PCBs. A framework has been presented by Vafeiadis et al to differentiate between various classifiers such as SVM which uses machine learning to detect the defects in PCBs. The results prove that the Support Vector Machine (SVM) classifier had the best accuracy [13].

\section{METHODOLOGY}

A PCB test system is developed in this study to detect PCB bias involving five stages. Categories image capture, image deletion, blocking, filtering, and feature detection.The Fig 1. Shows the PCB fault detection algorithm.

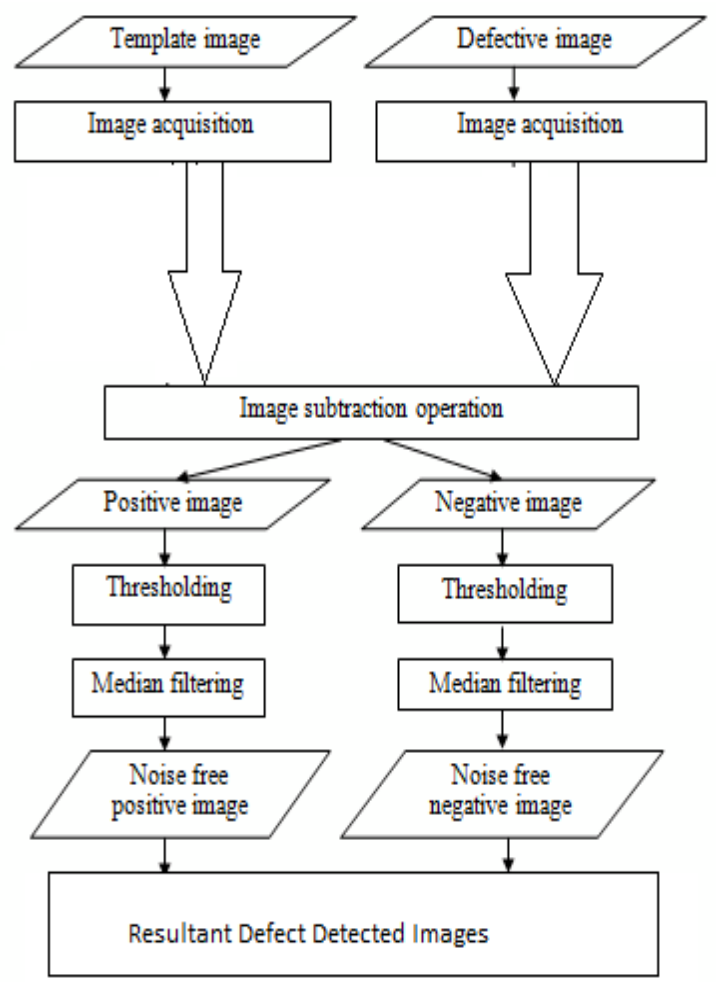

Fig 1. Block diagram of proposed PCB fault detection system

\subsection{Image acquisition:}

A picture of a printed Print Circuit Board is uploaded to a computer. While photographing the appropriate light source is provided and then uploaded to a computer. This step means getting an image that will be checked for errors in various image formats such as bitmap (.bmp), portable network graphics (.png), JPEG (.jpg), etc. a flawless PCB is considered an input that can also be the same or different image format.

\subsection{Image Subtraction:}

After the image is found the next step is to remove the image which means removing the wrong image from the template image. Extraction is done in pixel with pixel template and corrupted image.

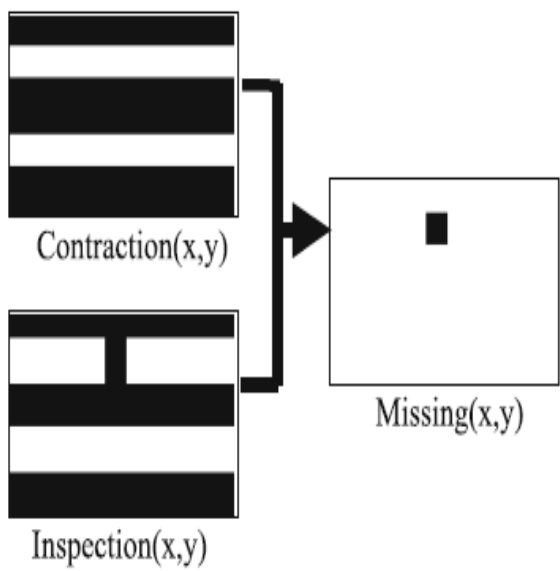

Fig. 2. Image subtraction operation of missing pixel

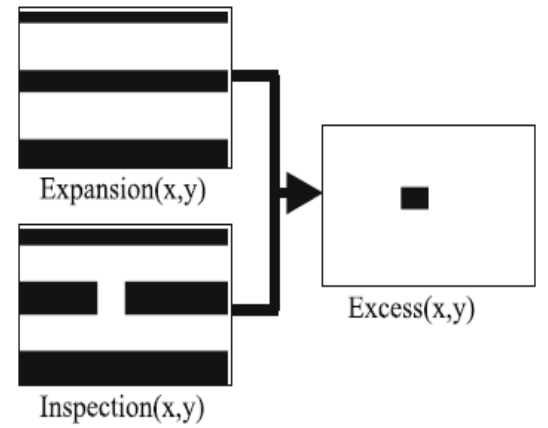

Fig. 3. Image subtraction operation of excess pixel

\subsection{Thresholding}

After selection of errors Images are Threshold in size and shape. Thresholding makes the pixel below Threshold value zero i.e. black and pixel with a value above the limit value white. This function therefore makes it easier for PCB tracks to appear more visually in the image. The test image and the template image are given the same limitation value to minimize system complexity.

\subsection{Filtering}

Smooth, also called blurring, is a process of image processing used to reduce the noise in an image to produce a pixel-based and clear image. The most common type of filter is the direct filter as the central filter used in our proposed system. This filter is used to reduce unreasonable noise or salt and pepper in a photo by keeping useful features and edges of the image. Median filtering is an offline process where the output of the processed pixel is obtained by calculating the pixel window dimensions around that pixel being read.

\subsection{Defect Detection}

Errors appear as white spots in the resulting image due to image capture function. If there are no spots on the image then the PCB is flawless. If there are spots on the resulting image then there is a defect in the image. The classification of errors based on the resulting image will be included in the next part of the work.

\section{RESULTS}

The performance of the proposed work is validated in this section. The simulation is carried out using MATLAB 2017b. Various test images along with their sets of master images have been used here to check the performance of the proposed system by simulating the proposed. collected 100 images with a resolution of $1024 * 1024$, which includes multiple defects in them. Since this high resolution may cause a large amount of 
computation, each defect and template picture is resized them into $256 * 256$

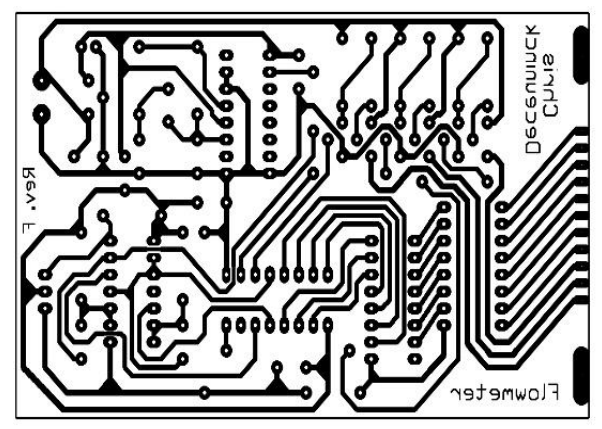

Fig.4. Master PCB Image 1 (G1)

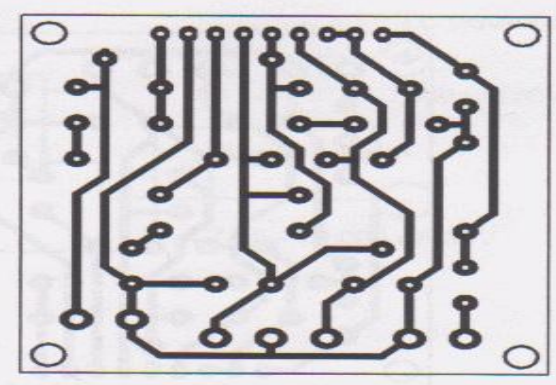

Fig.5. Master PCB Image 2(G2)

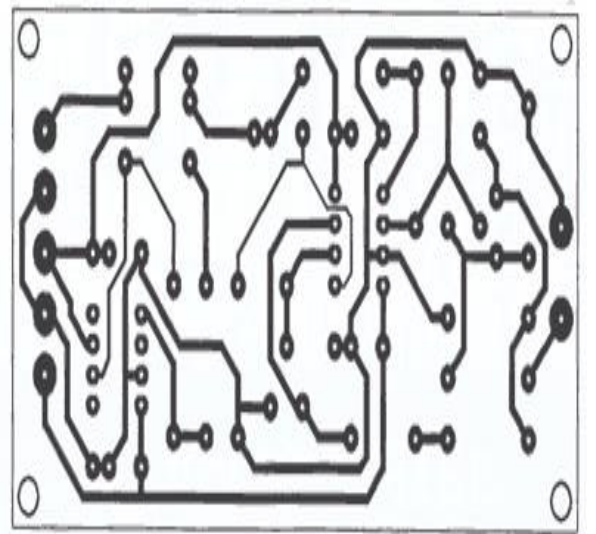

Fig.6. Master PCB Image 3 (G3)

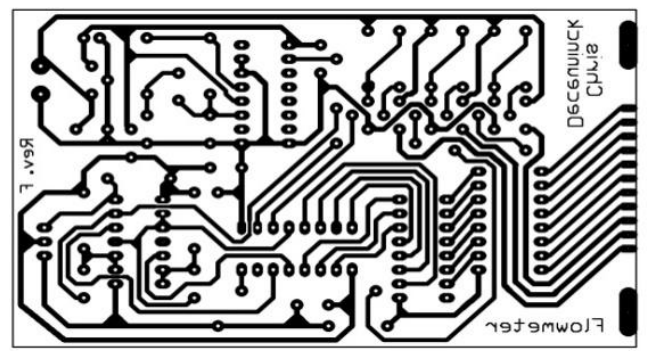

Fig 7: Missing Pin hole defect of Master PCB Image1

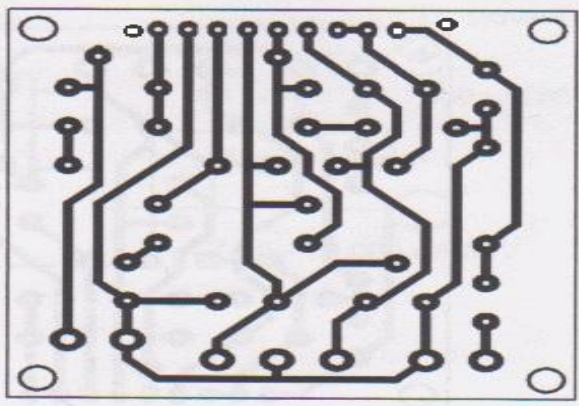

Fig 8: Pin Hole defect of Master PCB Image2

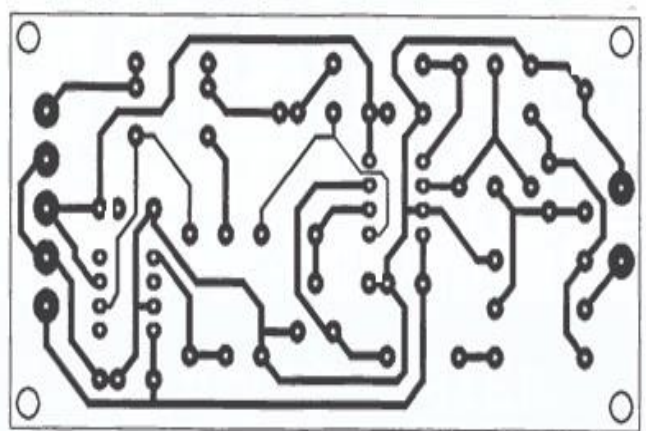

Fig 9: Open circuit defect of Master PCB Image 3

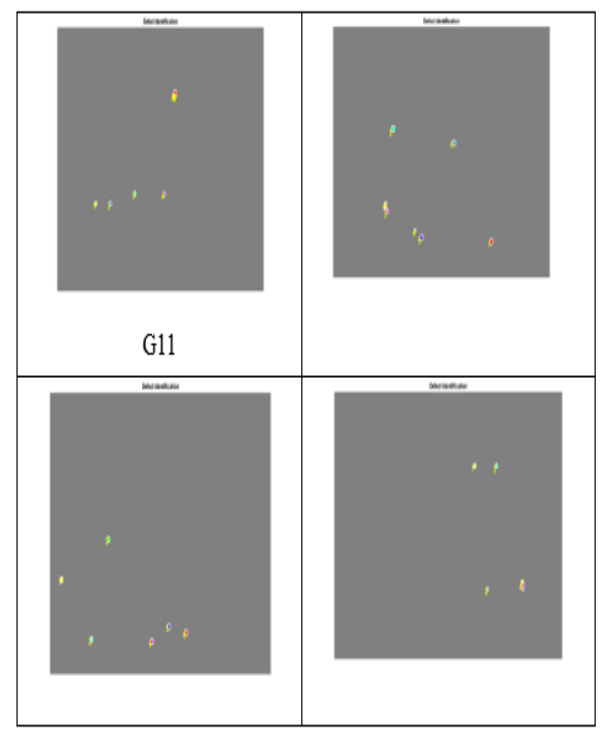

Fig.10. Detection of defects for master PCB image 1 


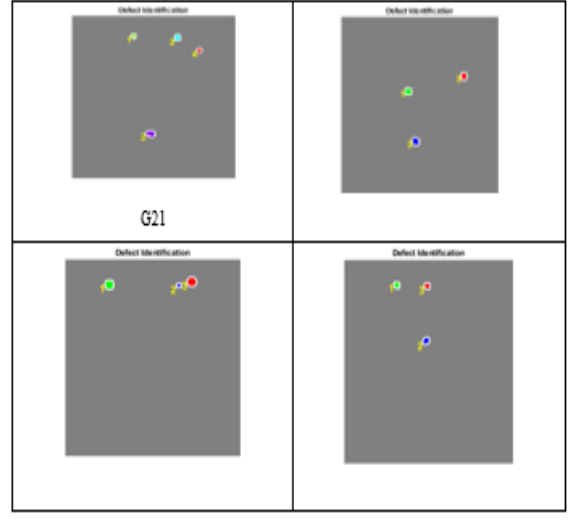

Fig.11. Detection of defects for master PCB image 2

The above images is result of image subtraction of the template and defective PCB Image. The numbers on the Image depict that some defects is present in the Image. The defects are may be missing hole, open circuit, short ciruit, mouse bite, spur, etc. The proposed method effectively detect the different defects present in the test image and numbering it and also achievs fast processing due to efective usage of image subtraction method.

Table 2. Classified Defect groups

\begin{tabular}{|c|c|c|}
\hline Number & Image & Defect classified \\
\hline 1 & G11 & Missing pin hole \\
\hline 2 & G21 & Wrong size hole \\
\hline 3 & G31 & Open circuit \\
\hline
\end{tabular}

The defects classified by these groups are listed in Table 2. For this particular exercise, each group is able to classify a minimum of 1 defect to a maximum of 4 defects, and is able to improve the image processing algorithm.

\subsection{Accuracy}

Accuracy measures the number of samples correctly classified cent of all proportion the number of samples. Accuracy for proposed method tabulated in Table 3 and gives $100 \%$ accuracy rate which means proposed method can capable of detecting any type of defect present in the PCB image.

Table 3. Accuracy of Proposed defect detection system

\begin{tabular}{|c|c|c|}
\hline Number & Images/Dataset & Accuracy (\%) \\
\hline 1 & 20 & $100 \%$ \\
\hline 2 & 30 & $100 \%$ \\
\hline 3 & 40 & $100 \%$ \\
\hline
\end{tabular}

\section{CONCLUSION}

This paper analyzes the output and features that the PCB system receives using image processing method. The program consists of image detection, image deletion, image blocking, and image filtering following error detection process. MATLAB-compiled image processing analysis gives the strong base for online access and speed of PCB quality. This proposed technique can achieve very high performance and measurement accuracy. Future enhancement for the algorithm should include the ability to classify all 14 defects individually. Integration with an image capturing system such as camera, frame grabber and personal computer is also essential for actual performance verification of defect detection and classification of PCBs.

\section{REFERENCES}

[1] Bing Hu And Jianhui Wang "Detection of PCB Surface Defects With Improved Faster-RCNN and Feature Pyramid Network" IEEE june 2020

[2] Venkat Anil Adibhatla etal "Defect Detection in Printed Circuit Boards Using You-Only-Look-Once Convolutional Neural Networks" mdpi journal Electronics Aug 2020

[3] Saeed Khalilian etal "PCB Defect Detection Using Denoising Convolutional Auto encoders" arXiv:2008.12589 [eess.IV] 2020.

[4] K. Kamalpreet and K. Beant, "PCB Defect Detection and Classification Using Image Processing," International Journal of Emerging Researchin Management \& Technology, vol. 03, 2014.

[5] W.-Y. Wu, M.-J. J. Wang and C.-M. Liu, "Automated inspection of printed circuit boards through machine vision," Computers in Industryvol. 28, no. 2, pp. 103-111, 1996.

[6] P. Wei, C. Liu , M. Liu, Y. Gao and H. Liu, "CNN-based reference comparison method for classifying bare $\mathrm{PCB}$ defects," The Journal of Engineering, vol. 2018, no. 16, pp. 1528-1533, 2018.

[7] A. K. P, S. N. S and S. . K. V. V, "A review of PCB defect detection using image processing," International Journal of Engineering and Innovative Technology (IJEIT), vol. 4, no. 11, pp. 188-192, 2015.

[8] N. Dave, V. Tambade and B. Pandhare, "PCB Defect Detection Using Image Processing And Embedded System," International Research Journal of Engineering and Technology (IRJET), vol. 3, no. 5, pp. 1897- 1901, 2016.

[9] S. H. I. Putera and Z. Ibrahim, "Printed circuit board defect detection using mathematical morphology and MATLAB image processing tools," in 2010 2nd International Conference on Education Technology and Computer, Shanghai, China, IEEE, 2010.

[10] Z. Ibrahim, S. A. R. Al-Attas, Z. Aspar and M. M. Mokji, "Performanc evaluation of wavelet-based PCB defect detection and localization algorithm," in 2002 IEEE International Conference on Industrial Technology 2002. IEEE ICIT '02., Bankok, Thailand, Thailand, IEEE, 2002.

[11] P. K. Srimani and V. Prathiba, "Adaptive data mining approach for PCB defect detection and classification," Indian Journal of Science and Technology, vol. 9, no. 44, pp. 1-9, 2016.

[12] A. Kusiak and C. Kurasek, "Data mining of printed-circuit board defects," IEEE Transactions on Robotics and Automation, vol. 17, no. 2, pp. 191-196, 2001.

[13] T. Vafeiadis, N. Dimitriou, D. Ioannidis, T. Wotherspoon, G. Tinkerand D. Tzovaras, "A framework for inspection of dies attachment on PCB utilizing machine learning techniques," Journal of Management Analytics, vol. 5, no. 2, pp. 81-94, 2018.

[14] V. A. Adibhatla, J.-S. Shieh, M. F. Abbod, H.-C. Chih, C.C. Hsu and J. Cheng, "Detecting Defects in PCB using Deep Learning via Convolution Neural Networks," in 2018 13th International Microsystems, Packaging, Assembly and Circuits Technology Conference (IMPACT), Taipei, Taiwan, Taiwan, IEEE, 2018, pp. 202-205. 
[15] C. Zhang, W. Shi, X. Li, H. Zhang and H. Liu, 'Improved bare PCB defect detection approach based on deep feature learning," The Journal of Engineering, vol. 2018, no. 16, pp. 1415-1420, 2018.

[16] Y.-S. Deng, A.-C. Luo and M.-J. Dai, "Building an Automatic Defect Verification System Using Deep Neural Network for PCB Defect Classification," in 2018 4th International Conference on Frontiers of Signal Processing (ICFSP), Poitiers, France, IEEE, 2018.

[17] L. Zhang, Y. Jin, X. Yang, X. Li, X. Duan, Y. Sun and H. Liu, "Convolutional neural network-based multi-label classification of PCB defects," The Journal of Engineering, vol. 2018, no. 16, pp. 1612-1616, 2018.

[18] S. Tang, F. He, X. Huang and J. Yang, "Online PCB Defect Detector On A New PCB Defect Dataset," arXiv preprint arXiv:1902.06197, 2019.

[19] A. Mujeeb, W. Dai, M. Erdt and A. Sourin, "One class based feature learning approach for defect detection using deep autoencoders," Advanced Engineering Informatics, vol. 42, 2019 